\title{
EXISTENCE OF CONDITIONALLY PERIODIC ORBITS FOR THE MOTION OF A SATELLITE AROUND THE OBLATE EARTH*
}

\author{
BY \\ RICHARD BARRAR \\ System Development Corporation, Santa Monica, California
}

\begin{abstract}
The author's previous results on the convergence of the Poincare-von Zeipel procedure in celestial mechanics are applied to the problem of the motion of a satellite around the oblate earth. The investigation concerns a potential that can vary both longitudinally and latitudinally, and also includes behavior near the critical angle. The existence of conditionally periodic orbits in all these cases is established.

Introduction. This paper shows the existence of conditionally periodic orbits for the motion of a satellite around the oblate earth.

W. T. Kyner [10] and C. Conley [4] have treated this question on the basis of the Moser [11] theorem on invariant curves of an area-preserving mapping of an annulus onto itself.

The method of proof of the present paper is based on Barrar [1], which proves the convergence of the Poincaré-von Zeipel procedure. This permits the investigation of a potential that can vary both longitudinally and latitudinally, and the investigation of the behavior near the critical inclination.

Moreover, although the approach is different, the present paper offers a mathematical justification for the procedure used by Brouwer in [2] away from the critical angle and by Hori in [7] near the critical angle; at least for sufficiently small $J_{2}$.

In the present paper we wish to apply the theorem proved in [1] to the oblate earth problem. The theorem has the following form for two degrees of freedom and there is no difficulty in extending it to $n$ degrees of freedom.

Existence Theorem 1. Consider a Hamiltonian of the form:
\end{abstract}

$$
H=H_{0}\left(p_{1}\right)+\mu\left[H_{1}\left(p_{1}, p_{2}\right)+H_{2}\left(p_{1}, p_{2}, q_{1}, q_{2}\right)\right] \quad \text { with } 0<\mu \leq 1,
$$

and corresponding differential equations

$$
\frac{d p_{i}}{d t}=\frac{\partial H}{\partial q_{i}}, \quad \frac{d q_{i}}{d t}=-\frac{\partial H}{\partial p_{i}}, \quad i=1,2,
$$

where all functions are assumed to be analytic, and $H_{2}$ is periodic of period $2 \pi$ in $q_{1}$ and $q_{2}$.

Now for two fixed values $p_{1}^{0}$ and $p_{2}^{0}$, let

$$
\lambda_{1}=\left.\frac{\partial\left(H_{0}+\mu H_{1}\right)}{\partial p_{1}}\right|_{D_{i}^{\circ}}, \quad \lambda_{2}=\left.\frac{\partial H_{1}}{\partial p_{2}}\right|_{p_{i}} .
$$

satisfy, ${ }^{* *}$ for some $\delta>0$,

$$
\left|n_{1}+n_{2} \frac{\mu \lambda_{2}}{\lambda_{1}}\right| \geq \frac{\delta \mu}{\left(n_{2}\right)^{2}} \quad \text { for all integers } n_{1} \text { and } n_{2} \text { with } n_{2} \neq 0 \text {. }
$$

*Received March 3, 1965; revised manuscript received July 21, 1965.

${ }^{* *}$ For $0 \leq\left(\lambda_{2} / \lambda_{1}\right) \leq M$ the exterior measure of all $\left(\lambda_{2} / \lambda_{1}\right)$ not satisfying (H3) is less than $\left(4 \pi^{2} / 3\right) M \mu \delta$ (see Siegel [13]). 
Further, for all $\mu$ in some neighborhood of $\mu_{0}$, with $0 \leq \mu_{0} \leq 1$, let

$$
\operatorname{det}\left|\frac{\partial^{2}\left(H_{n}+\mu H_{1}\right)}{\partial p_{i} \partial p_{i}}\right|_{D_{i} \circ}=2 N \mu \neq 0 .
$$

Then for $\left|H_{2}\right| \leq \epsilon(\delta, N)$, and for $\mu$ in the above neighborhood, there is a conditionally periodic solution of (1) of the form:

$$
\begin{aligned}
& q_{1}=\lambda_{1}\left(t-\tau_{1}\right)+\phi_{1}\left(e^{i \lambda_{1} t}, e^{i \mu \lambda_{2} t}\right), \\
& q_{2}=\mu \lambda_{2}\left(t-\tau_{2}\right)+\phi_{2}\left(e^{i \lambda_{2} t}, e^{i \mu \lambda_{2} t}\right), \\
& p_{i}=A_{i}+\psi_{i}\left(e^{i \lambda_{1} t}, e^{i \mu \lambda_{2} t}\right),
\end{aligned}
$$

where $A_{i}$ and $\tau_{i}$ are constants.

The oblate earth problem. In terms of suitably chosen units, the gravitational potential of the earth may be put in the form:

$$
V=-\frac{1}{r}\left[1-\sum_{p=2}^{\infty} J_{p} \frac{1}{r^{p}} P_{p}(\cos \theta)-\sum_{p=2}^{\infty} \sum_{\substack{m=-p \\ m \neq 0}}^{\nu} J_{p m} \frac{1}{r^{p}} P_{p}^{m}(\cos \theta) e^{i m \phi}\right]
$$

where the unit of length is the earth's equatorial radius, and $r, \theta$ and $\phi$ are standard spherical coordinates; $J_{p}$ and $J_{p m}$ are constants determined by the mass and the shape of the earth; and $P_{p}$ and $P_{p}^{m}$ are spherical harmonics.

Since it is assumed that this development is about the earth's center of mass, one sets $J_{1} \equiv 0, J_{1 m} \equiv 0$.

It has been found experimentally that $J_{2} \sim 1 \times 10^{-3}$ and the sum of all the other terms in the infinite sum is $\sim 1 \times 10^{-6}$.

We shall begin our investigations with a discussion of the motion resulting from the potential

$$
V=-\frac{1}{r}+\frac{J_{2} P_{2}(\cos \theta)}{r^{3}}+\frac{J_{4} P_{4}(\cos \theta)}{r^{5}},
$$

because this potential brings out all the essential features of the problem; later we will discuss the general potential (1). For convenience, let us set $J_{2}=\epsilon$; then $J_{4}=O\left(\epsilon^{2}\right)$.

In terms of the standard Delaunay canonical variables,

$$
\begin{array}{lll}
p_{1}=\sqrt{a} & (a=\text { semi-major axis }), & q_{1}=\text { mean anomaly }, \\
p_{2}=p_{1}\left(1-e^{2}\right)^{1 / 2} & (e=\text { eccentricity }), & q_{2}=\text { argument of perigee }, \\
p_{3}=p_{2} \cos I & (I=\text { inclination }), & q_{3}=\text { longitude of ascending node },
\end{array}
$$

and using the spherical trigonometric identity $\cos \theta=\sin I \sin \left(q_{2}+f\right)$ with $f$ the true anomaly, the Hamiltonian $H$ for the potential (2) has the form:*

$$
\begin{gathered}
H=F_{0}\left(p_{1}\right)+\epsilon\left[F_{1}\left(p_{1}, p_{2}, p_{3}\right)+F_{2}\left(p_{1}, p_{2}, p_{3}, q_{1}, q_{2}\right)\right]+\epsilon^{2} F\left(p_{1}, p_{2}, p_{3}, q_{1}, q_{2}\right), \\
F_{0}\left(p_{1}\right)=\frac{1}{2 p_{1}^{2}},
\end{gathered}
$$

*In Eq. (3) and throughout the remainder of this paper we use the notation that $F\left(p_{1}, p_{2}, p_{3}, q_{1}, q_{2}\right)$ is an analytic function of all its variables, and is periodic of period $2 \pi$ in $q_{1}$ and $q_{2}$. 


$$
\begin{aligned}
F_{1}\left(p_{1}, p_{2}, p_{3}\right)= & \frac{1}{2\left(p_{1} p_{2}\right)^{3}}\left[-\frac{1}{2}+\frac{3}{2}\left(\frac{p_{3}}{p_{2}}\right)^{2}\right] \\
F_{2}\left(p_{1}, p_{2}, p_{3}, q_{1}, q_{2}\right)= & \frac{1}{2\left(p_{1}\right)^{6}}\left[-\frac{1}{2}+\frac{3}{2}\left(\frac{p_{3}}{p_{2}}\right)^{2}\right]\left[\left(\frac{a}{r}\right)^{3}-\left(\frac{p_{1}}{p_{2}}\right)^{3}\right] \\
& +\frac{1}{2\left(p_{1}\right)^{6}}\left[\frac{3}{2}-\frac{3}{2}\left(\frac{p_{3}}{p_{2}}\right)^{2}\right]\left(\frac{a}{r}\right)^{3} \cos \left(2 q_{2}+2 f\right) \\
= & \sum_{n_{1} \neq 0} A_{n_{1}}\left(p_{1}, p_{2}, p_{3}\right) \cos \left(n_{1} q_{1}\right)+B_{n_{2}}\left(p_{1}, p_{2}, p_{3}\right) \cos \left(n_{1} q_{1}+2 q_{2}\right) .
\end{aligned}
$$

For details, see Brouwer [2] or Brouwer and Clemence [3, pp. 562-573]. The general method of introducing the Delaunay canonical variables into a problem with a potential of the form (1) or (2) is developed in Smart [13, Chapters 9-11], and Brouwer and Clemence [3, Chapter XI, §9]. In (3), we have explicitly written out only the perturbation term $J_{2} P_{2}(\cos \theta) / r^{3}$.

Since

$$
e=\left(1-\left(\frac{p_{2}}{p_{1}}\right)^{2}\right)^{1 / 2}, \quad \cos I=\frac{p_{3}}{p_{2}},
$$

it follows that for the motion to be bounded with $0<e<1,-\pi<I<\pi$, we must restrict ourselves to regions where

$$
0<p_{3}<p_{2}<p_{1} .
$$

From the identity resulting from conservation of angular momentum,

$$
\frac{\partial f}{\partial q_{1}}=\left(\frac{p_{2}}{p_{1}}\right)\left(\frac{a}{r}\right)^{2}=\left(\frac{p_{1}}{p_{2}}\right)^{3}(1+e \cos f)^{2},
$$

it readily follows that $\left(f-q_{1}\right)$ is an analytic and periodic function of $q_{1}$ in a strip in the complex $q_{1}$-plane surrounding the real $q_{1}$-axis with the width of the strip depending on $e$. By the equation of an ellipse in polar coordinates,

$$
\frac{a}{r}=\left(\frac{p_{1}}{p_{2}}\right)^{2}(1+e \cos f)
$$

this also applies to $(a / r)$. The actual development of $\cos f$ and $\sin f$ in terms of $\cos q_{1}$ and $\sin q_{1}$ are well known (see Smart [13, p. 41]). From these remarks, we deduce the validity of the expansion in (3). It is shown in Brouwer [2, p. 379] that no term $n_{1}=0$ appears in the expansion of $F_{2}$.

Thus, if we stay in neighborhoods

$$
\begin{aligned}
N_{0}: \quad & \left|p_{i}-p_{i}^{0}\right|<a_{i} \\
& 1-\xi_{0}<\left|e^{i a_{i}}\right|<1+\xi_{0}
\end{aligned}
$$

for sufficiently small $a_{i}, \xi_{0}$; and the constants $p_{i}^{0}$ satisfying (5); all functions will be analytic. We assume this to be always fulfilled in what follows.

For completeness we remark that if $\psi=\psi\left(p_{1}, p_{2}, p_{3}, q_{1}, q_{2}\right)=\psi\left(a, e, I, q_{1}, q_{2}\right)$, one has the identities:

$$
\frac{\partial \psi}{\partial p_{1}}=\frac{1}{e} \frac{\partial \psi}{\partial e} \frac{\left(p_{2}\right)^{2}}{\left(p_{1}\right)^{3}}, \quad \frac{\partial \psi}{\partial p_{2}}=-\frac{1}{e} \frac{\partial \psi}{\partial e} \frac{p_{2}}{\left(p_{1}\right)^{2}} .
$$


Moreover, in the term $F_{2}$ of the Hamiltonian (3) one has for small $e$ the readily established expansions:

$$
\left(\frac{a}{r}\right)^{3}=\left(1+3 e \cos q_{1}\right)+O\left(e^{2}\right)
$$

and

$$
\cos \left(2 q_{2}+2 f\right)=\cos \left(2 q_{1}+2 q_{2}\right)-4 e \sin \left(2 q_{1}+2 q_{2}\right) \sin q_{1}+O\left(e^{2}\right) .
$$

Hence, it follows from (8) and (9) that as $e \rightarrow 0$, the canonical differential equations (H2) become meaningless. For this reason, we must restrict to values of $e$ bounded away from zero.

It should be mentioned, however, that Poincaré has developed methods for dealing with this type of difficulty. More is required than the mere introduction of Poincaré variables, because with Poincaré variables the Hamiltonian is no longer of the correct form for our Existence Theorem 1 to apply. Rather, using the method developed in Poincaré [12, Chapter XII], it can be shown that if one makes a canonical transformation using the so-called Poincaré periodic orbits of the first kind, the singularity at $e=0$ may be removed. We hope to return to this point in a future paper. In the present paper, we shall always assume that $e$ is bounded away from zero.

Since we have restricted to the potential (2), the variable $q_{3}$ does not enter into the development (3). It then follows from the canonical differential equations (H2) that $p_{3}$ is a constant. Thus, we consider only the four variables $p_{1}, p_{2}, q_{1}$ and $q_{2}$. In a later section of the paper we shall indicate the changes that should be made to consider also the variables $p_{3}$ and $q_{3}$ and the more general potential (1).

Existence of conditionally periodic orbits away from the critical angle. We wish now to transform the Hamiltonian (3) to the form:

$$
H=F_{0}\left(P_{1}\right)+\epsilon F_{1}\left(P_{1}, P_{2}\right)+\epsilon^{2} F\left(P_{1}, P_{2}, Q_{1}, Q_{2}, \epsilon\right)
$$

so that we can apply our Existence Theorem 1 on conditionally periodic orbits.

In essence this means that we want to get rid of the term $\epsilon F_{2}$ of (3) and replace it by something of order $O\left(\epsilon^{2}\right)$. It was for just this purpose that the Poincaré-von Zeipel transformation was developed (see Poincaré [12, \$125]). Since $F_{2}$ contains no term $n_{1}=0$, the transformation reduces to the following canonical transformation from $(p, q)$ to $(P, Q)$ by means of the generating function $W$ :

$$
W=P_{1} q_{1}+P_{2} q_{2}+\epsilon S\left(P_{1}, P_{2}, q_{1}, q_{2}\right)
$$

with

$$
\begin{aligned}
& S=\left(P_{1}\right)^{3} \int F_{2}\left(P_{1}, P_{2}, q_{1}, q_{2}\right) d q_{1} \text { or } \frac{\partial F_{0}\left(P_{1}\right)}{\partial P_{1}} \frac{\partial S}{\partial q_{1}}+F_{2}\left(P_{i}, q_{i}\right)=0, \\
& p_{i}=P_{i}+\epsilon \frac{\partial S}{\partial q_{i}}, \quad Q_{i}=q_{i}+\epsilon \frac{\partial S}{\partial P_{i}}, \quad i=1,2 .
\end{aligned}
$$

Brouwer [2] has explicitly calculated $S$. It is clearly an analytic function of all its variables, and periodic of period $2 \pi$ in $q_{1}$ and $q_{2}$. The rationale for the introduction of $S$ is indicated in the discussion below (12).

It now follows, by methods exactly analogous to our proof of Lemma 1 of [1], that 
for sufficiently small $\epsilon$, the transformation $W$ may be written as

$$
p_{i}=P_{i}+L_{i}(P, Q), \quad q_{i}=Q_{i}+K_{i}(P, Q),
$$

where $L_{i}$ and $K_{i}$ are periodic of period $2 \pi$ in $Q_{1}$ and $Q_{2}$; it also follows that $L_{i}$ and $K_{i}$ are defined in a region

$$
N_{1}:\left|P_{i}-P_{i}^{0}\right|<b_{i}, \quad 1-\xi_{1}<\left|e^{i Q_{i}}\right|<1+\xi_{1},
$$

and that they map this region $N_{1}$ into the region $N_{0}$ of (7). Hence from now on we may restrict ourselves to the region $N_{1}$. In $N_{1}$ the Hamiltonian has the form:

$$
\begin{aligned}
H= & F_{0}\left(P_{1}+\epsilon \frac{\partial S}{\partial q_{1}}\right)+\epsilon\left[F_{1}\left(P_{i}+\epsilon \frac{\partial S}{\partial q_{i}}\right)+F_{2}\left(P_{i}+\epsilon \frac{\partial S}{\partial q_{i}}, q_{i}\right)\right]+\epsilon^{2} F\left(p_{1}, p_{2}, q_{1}, q_{2}\right) \\
= & F_{0}\left(P_{1}\right)+\epsilon F_{1}\left(P_{1}, P_{2}\right)+\epsilon\left[\frac{\partial F_{0}\left(P_{1}\right)}{\partial P_{1}} \frac{\partial S}{\partial q_{1}}+F_{2}\left(P_{i}, q_{i}\right)\right] \\
& +\epsilon^{2}\left[\frac{1}{2} \frac{\partial^{2} F_{0}\left(P_{1}+\theta \frac{\partial S}{\partial q_{1}}\right)}{\partial P_{1}^{2}}\left(\frac{\partial S}{\partial q_{1}}\right)^{2}+\frac{\partial F_{1}\left(P+\theta \frac{\partial S}{\partial q}\right)}{\partial P_{i}}\left(\frac{\partial S}{\partial q_{i}}\right)+\frac{\partial F_{2}\left(P+\theta \frac{\partial S}{\partial q}, q\right)}{\partial P_{i}}\left(\frac{\partial S}{\partial q_{i}}\right)\right] \\
& +\epsilon^{2} F\left(p_{1}, p_{2}, q_{1}, q_{2}\right), \quad 0 \leq \theta \leq \epsilon .
\end{aligned}
$$

(11) shows that the second term in $\epsilon$ in (12) vanishes, and the discussion above shows that the coefficients of all terms in $\epsilon^{2}$ and higher are bounded. Therefore, our assertion is established. With the Hamiltonian in the form (10), we can now apply Existence Theorem 1. Let

$\lambda_{1}=\frac{\partial\left(F_{0}+\epsilon F_{1}\right)}{\partial P_{1}}=-\frac{1}{P_{1}^{3}}-\frac{3}{2} \epsilon \frac{1}{P_{1}^{4} P_{2}^{3}}\left(-\frac{1}{2}+\frac{3}{2}\left(\frac{P_{3}}{P_{2}}\right)^{2}\right), \quad \epsilon \lambda_{2}=\frac{3}{4} \epsilon \frac{1}{P_{1}^{3} P_{2}^{4}}\left(1-5\left(\frac{P_{3}}{P_{2}}\right)^{2}\right)$.

Then for $P_{1}^{0}, P_{2}^{0}$ and $P_{3}^{0}$ that satisfy:

$$
\begin{aligned}
&\left|n_{1} \lambda_{1}+n_{2} \epsilon \lambda_{2}\right| \geq \frac{\delta \epsilon}{n_{2}^{2}}, \quad n_{1} \text { and } n_{2} \text { integers, } n_{2} \neq 0, \\
& \operatorname{det}\left|\frac{\partial^{2}\left(F_{0}+\epsilon F_{1}\right)}{\partial P_{i} \partial P_{i}}\right|_{P_{i} \bullet}=N \epsilon>0,
\end{aligned}
$$

and for sufficiently small $\epsilon$, it follows from Existence Theorem 1 that there will exist conditionally periodic solutions of the equations of motion corresponding to the Hamiltonian (3). These solutions will be of the form:

$$
\begin{array}{ll}
q_{1}=\lambda_{1}\left(t-\tau_{1}\right)+\phi_{1}\left(e^{i \lambda_{1} t}, e^{i \epsilon \lambda_{2} t}\right), & q_{2}=\epsilon \lambda_{2}\left(t-\tau_{2}\right)+\phi_{2}\left(e^{i \lambda_{1} t}, e^{i \epsilon \lambda_{2} t}\right), \\
p_{1}=A_{1}+\psi_{1}\left(e^{i \lambda_{1} t}, e^{i \epsilon \lambda_{2} t}\right), & p_{2}=A_{2}+\psi_{2}\left(e^{i \lambda_{1} t}, e^{i \epsilon \lambda_{2} t}\right) .
\end{array}
$$

Condition (B) can always be fulfilled even though, as the Hamiltonian is now written, the condition is not met at one point; namely, because $\partial^{2}\left(F_{0}+\epsilon F_{1}\right) / \partial P_{2} \partial P_{2}=$ $\left(3 \epsilon / 2 P_{1}^{3} P_{2}^{5}\right)\left(2-15\left(P_{3} / P_{2}\right)^{2}\right)$, condition (B) is not met at $\cos ^{2} I=\frac{2}{15}$. However, by squaring the Hamiltonian (as suggested by Poincare $[12, \S 43]$ for a slightly different case), and considering the resulting equivalent Hamiltonian at $\cos ^{2} I=\frac{2}{15}$, it is readily found that condition $(B)$ is now met.

However, condition (A) cannot always be fulfilled. At $\cos ^{2} I=\frac{1}{5}$, the frequency $\lambda_{2} \equiv 0$, so Existence Theorem 1 no longer applies. This is the so-called critical inclination. 
The next section of this paper is devoted to studying the phenomena very near this angle. However, the discussion above shows that away from the critical inclination the assumptions of Existence Theorem 1 are met, assuring conditionally periodic orbits for almost all frequencies, for small enough $\epsilon$.

The critical inclination angle. We now wish to introduce a series of canonical transformations that will transform the Hamiltonian near the critical inclination angle into a form where Existence Theorem 1 may be applied.

The motivation for this series of transformations is found in Poincaré [12, §206]. (For a discussion of the involved physical phenomena that occur near the critical angle, the reader is referred to Poincaré $[12, \S \S 199,200]$ and Hagihara [6].)

In essence, the series of canonical transformations that we now introduce, replaces the old canonical variables by a new set of canonical variables that are uniformizing in a sense made clear below. At the present state in our development the Hamiltonian (10) has the form:

$$
\begin{array}{r}
H=F_{0}\left(p_{1}\right)+\epsilon F_{1}\left(p_{1}, p_{2}\right)+\epsilon^{2}\left[F_{21}\left(p_{1}, p_{2}\right)+F_{22}\left(p_{1}, p_{2}\right) \cos 2 q_{2}+F_{23}\left(p_{1}, p_{2}, q_{1}, q_{2}\right)\right] \\
+\epsilon^{3} F\left(p_{1}, p_{2}, q_{1}, q_{2}, \epsilon\right),
\end{array}
$$

where we have changed $P$ and $Q$ back to $p$ and $q$. The exact form of the terms $F_{0}, F_{1}, F_{21}$ and $F_{22}$ are given in Hori [7, Eq. (6)].

Let $p_{i}^{0}$ be fixed values at the critical angle $\cos ^{2} I=\frac{1}{5}$. At this angle it follows from the form of $F_{0}$ and $F_{1}$ that the following equation holds:

$$
\begin{aligned}
& \left.\frac{\partial F_{0}}{\partial p_{1}}\right|_{p^{\circ} \circ}=m_{2} \neq 0,\left.\frac{\partial\left(F_{0}+\epsilon F_{1}\right)}{\partial p_{2}}\right|_{p_{i}^{\circ}}=0,\left.\quad \frac{\partial F_{1}}{\partial p_{1}}\right|_{p^{\circ}}=\lambda_{1} \neq 0, \\
& \left.\frac{\partial^{2} F_{1}}{\partial p_{i} \partial p_{i}}\right|_{p^{\circ} \circ}=\left(\begin{array}{cc}
A_{11} & 0 \\
0 & A_{22}
\end{array}\right), \quad A_{11}, A_{22} \text { not vanishing. }
\end{aligned}
$$

The canonical transformations. To remove the difficulty caused by $\lambda_{2}=0$, we now introduce a series of three canonical transformations, of which only the first is non-trivial.

The first transformation $p, q \rightarrow u, v$ is accomplished by means of the generating function:

$$
\begin{aligned}
W & =p_{1}^{0} q_{1}+p_{2}^{0} q_{2}+\sqrt{ } \epsilon T_{1}+\epsilon^{2} T_{2}, \\
T_{1} & =u_{1} q_{1}+\int d q_{2}\left(u_{2}-\psi\right)^{1 / 2}, \\
T_{2} & =-\frac{1}{m_{2}} \int d q_{1} F_{23}\left(p_{1}^{0}, p_{2}^{0}, q_{1}, q_{2}\right)
\end{aligned}
$$

with $\psi$ being defined by

$$
\frac{1}{2} A_{22} \psi=F_{21}+F_{22} \cos 2 q_{2},
$$

where we abbreviate

$$
F_{21}=F_{21}\left(p_{1}^{0}, p_{2}^{0}\right), \quad F_{22}=F_{22}\left(p_{1}^{0}, p_{2}^{0}\right) .
$$

Although the details are rather messy, by imitating our procedure in the expansion of Eq. (12), though now expanding in powers of $\sqrt{ } \epsilon$, we can proceed in a straight- 
forward fashion to find that in terms of the new canonical variables, the Hamiltonian is of the form:

$$
H=F_{0}\left(p_{1}^{0}\right)+\sqrt{\epsilon} \epsilon H^{*}
$$

with

$$
H^{*}=G_{1}\left(u_{1}\right)+\epsilon^{3 / 2} \frac{1}{2} A_{22} u_{2}+\epsilon^{2} F\left(u_{1}, u_{2}, q_{1}, q_{2}, \epsilon\right)
$$

and

$$
G_{1}\left(u_{1}\right)=m_{1} u_{1}+O(\sqrt{\epsilon}) .
$$

Further, in terms of the new Hamiltonian, $\partial H / \partial u_{2}=\epsilon^{3 / 2} A_{22} / 2 \neq 0$. This means the critical angle difficulty has disappeared. However, $H$ is not periodic of period $2 \pi$ in the angular variables $v_{1}$ and $v_{2}$ as follows from the change of variable equations:

$$
v_{1}=\frac{\partial W}{\partial q_{1}}=\sqrt{ } \epsilon q_{1}, \quad v_{2}=\frac{\partial W}{\partial u_{2}}=\frac{1}{2} \sqrt{ } \epsilon \int \frac{d q_{2}}{\left(u_{2}-\psi\right)^{1 / 2}} .
$$

The second transformation is rather trivial, it replaces $v_{i}$ by $z_{i}=v_{i} / \sqrt{ } \epsilon$. To see that this is canonical, note that since $F_{0}\left(p_{i}^{0}\right)$ is a constant, it follows from the canonical equations for $u_{i}$ and $v_{i}$ with respect to $H$, that $u_{i}$ and $z_{i}$ are canonical variables with respect to $H^{*}$.

Further, this second transformation replaces the second equation of (19) by the elliptic integral

$$
z_{2}=\frac{1}{2} \int \frac{d q_{2}}{\left(u_{2}-\psi\right)^{1 / 2}}
$$

Thus $q_{2}$ is an elliptic function of $z_{2}$. The parameter $k$ of the elliptic function depends on the constants $F_{21}$ and $F_{22}$ and on $u_{2}$. Let us merely summarize the various phenomena that can take place, when to a first approximation we assume $u_{2}$ is constant.

Case 1. $0 \leq k^{2}<1$.

Here $q_{2}$ increases by $2 \pi$ when $z_{2}$ increases by a period $P\left(u_{2}\right)$. This is the case that occurs away from the critical angle. A special subcase of this also occurs in the potential used by Vinti [16], for then $k^{2}=0$, as has been pointed out by Hori [7] and Izsak [8].

Case 2. $k^{2}>1$.

Here for real $z_{2}, q_{2}$ is bounded between two finite limits, and oscillates between them as $z_{2}$ continually increases. This is the phenomena of libration in celestial mechanics (see Hori [7], Garfinkel [5]) and also shows why we refer to $z_{2}$ as a uniformizing variable.

Case 3. $k^{2}<0$.

Here once again $q_{2}$ is a periodic function of $z_{2}$, with period $P\left(u_{2}\right)$. In the case of the oblate earth, if one considers only the term $J_{2} P_{2}(\sin \theta) / r^{3}$ and neglects $J_{4} P_{4}(\sin \theta) / r^{5}$, one is in Case 3, while if you do not neglect it you are in Case 2 with libration.

Finally, the case $k^{2}=1$ will be discussed below.

The third transformation is also trivial. It is introduced so that in terms of the final variables $P_{1}, P_{2}, Q_{1}$ and $Q_{2}$ the Hamiltonian will be of period $2 \pi$. It is

$$
\begin{array}{ll}
Q_{1}=z_{1}=q_{1}, & Q_{2}=\frac{2 \pi z_{2}}{P\left(u_{2}\right)}, \\
P_{1}=u_{1}, & P_{2}=\frac{1}{2 \pi} \int^{u_{s}} P(\xi) d \xi .
\end{array}
$$


It readily follows that this is a canonical transformation, and since $P(\xi)>0$, we also have $u_{2}=G_{2}\left(P_{2}\right)$ with $G_{2}^{\prime}\left(P_{2}\right) \neq 0$. Finally, the Hamiltonian $H^{*}$ in terms of the new variables is of the form

$$
H=G_{1}\left(P_{1}\right)+\epsilon^{3 / 2} \frac{1}{2} A_{22} G_{2}\left(P_{2}\right)+\epsilon^{2} F\left(P_{1}, P_{2}, Q_{1}, Q_{2}, \epsilon\right) .
$$

Thus in terms of the new variables all conditions of Existence Theorem 1 are fulfilled and we obtain the existence of conditionally periodic motions, for small enough $\epsilon$. By using the transformation formulas (16), (20), and (21), the motion in terms of $p_{i}$ and $q_{i}$ is of the form:

$$
\begin{aligned}
& q_{1}=\lambda_{1}(t-\tau)+h_{1}\left(e^{i \lambda_{1} t}, e^{i \epsilon=/ 2 \lambda_{z} t}\right), \\
& q_{2}=h_{2}\left(e^{i \lambda_{1} t}, e^{i \epsilon^{s} / \lambda_{z} t}\right), \\
& p_{i}=A_{i}+l_{i}\left(e^{i \lambda_{1} t}, e^{i \epsilon^{3} / \lambda_{z} t}\right)
\end{aligned}
$$

near the critical angle, which corresponds to libration.

There is one other case that occurs, which we have not considered. Namely; as $k^{2} \rightarrow 1$, the integral (20) approaches $\log \left(\tan \left(q_{2} / 2\right)+\pi / 4\right)$; this is equivalent to saying that the period $P\left(u_{2}\right)$ approaches infinity, and thus the transformation (21) is not defined. This case is exactly on the border line between the libration case and the usual case of revolution. In essence this corresponds to a critical angle in the new $\left(P_{1}, P_{2}\right.$, $\left.Q_{1}, Q_{2}\right)$ variables, since now $\partial G_{2}\left(P_{2}\right) / \partial P_{2}=0$.

Poincaré [12, $\$ 207-210$ and $\S \S 215-217]$ indicates how to treat this general situation. One defines a new canonical transformation by using the periodic solution that occurs at the critical angle in the new $\left(P_{1}, P_{2}, Q_{1}, Q_{2}\right)$ variables. The author hopes to return to this problem in a future paper.

Generalization. Let us now consider the general potential (1) and the resulting motion. Since $J_{2}$ is still the only term of $O(\epsilon)$, it is still valid to introduce the transformation (11) to obtain a Hamiltonian of the form:

$$
H=F_{0}\left(P_{1}\right)+\epsilon F_{1}\left(p_{1}, p_{2}, p_{3}\right)+\epsilon^{2} F\left(p_{1}, p_{2}, p_{3}, q_{1}, q_{2}, q_{3}, \epsilon\right) .
$$

It follows from (3) that $\partial F_{1} / \partial P_{3}=\lambda_{3}>0$ in the case of the oblate earth (corresponding to precession of the node). Hence, away from the critical angle, the three-degree-offreedom analogue of Existence Theorem 1 shows that for frequencies $\lambda_{1}, \epsilon \lambda_{2}, \epsilon \lambda_{3}$ such that

$$
\left|n_{1} \lambda_{1}+n_{1} \epsilon \lambda_{2}+n_{3} \epsilon \lambda_{3}\right| \geq \frac{\delta \epsilon}{\left(n_{1}^{2}+n_{2}^{2}+n_{3}^{2}\right)^{3 / 2}}
$$

there will be conditionally periodic orbits of the form:

$$
\begin{aligned}
& q_{1}=\lambda_{1}\left(t-\tau_{1}\right)+f_{1}\left(e^{i \lambda_{2} t}, e^{i \epsilon \lambda_{2} t}, e^{i \epsilon \lambda_{3} t}\right), \\
& q_{2}=\epsilon \lambda_{2}\left(t-\tau_{1}\right)+f_{2}\left(e^{i \lambda_{1} t}, e^{i \epsilon \lambda_{2} t}, e^{i \epsilon \lambda_{3} t}\right), \\
& q_{3}=\epsilon \lambda_{3}\left(t-\tau_{1}\right)+f_{3}\left(e^{i \lambda_{1} t}, e^{i \epsilon \lambda_{3} t}, e^{i \epsilon \lambda_{3} t}\right), \\
& p_{i}=A_{i}+g_{i}\left(e^{i \lambda_{1} t}, e^{i \epsilon \lambda_{3} t}, e^{i \epsilon \lambda_{3} t}\right) .
\end{aligned}
$$

At the critical inclination, $\lambda_{2}=0$; it therefore becomes necessary to study the terms of $O\left(\epsilon^{2}\right)$ in the equation corresponding to (14). In our previous discussion, where we consider only $J_{2}$ and $J_{4}$, we had terms of the form $C_{1}\left(P_{1}, P_{2}, P_{3}\right)$ and $C_{2}\left(P_{1}, P_{2}, P_{3}\right)$ 
$\cos 2 Q_{2}$. If one assumes there are other terms of $O\left(\epsilon^{2}\right)$ in (1), then additional terms must be considered at the critical angle. Thus when Kozai [9] also considered $J_{3}$ as $O\left(\epsilon^{2}\right)$, he obtained an additional term $C_{3}\left(P_{1}, P_{2}, P_{3}\right) \sin Q_{2}$. However, it follows from the general theory of elliptic integrals that this additional term $J_{3}$ will still result in an elliptic integral and thus offers no essential new difficulty. If other terms must also be considered, say, for example, $J_{5} \sim O\left(\epsilon^{2}\right)$, the problem becomes more complicated. The complete discussion would then involve the theory of the integration of algebraic functions on a Riemann surface, or hyperelliptic functions see, e.g., G. Springer [15]. However, at the time of writing of this paper, it is generally agreed that all terms besides $J_{2}, J_{3}$ and $J_{4}$ are insignificant. Overlooking this possible complication, we find by the methods used in this paper that libration takes place near the critical inclination and the orbits are of the form:

$$
\begin{aligned}
& y_{1}=\lambda_{1}(t-\tau)+h_{1}\left(e^{i \lambda_{2} t}, e^{i \epsilon / 2 \lambda_{3} t}, e^{i \epsilon \lambda_{3} t}\right), \\
& q_{2}=h_{2}\left(e^{i \lambda_{2} t}, e^{i \epsilon s / \lambda_{2} t}, e^{i \epsilon \lambda_{2} t}\right) \text {, } \\
& q_{3}=\epsilon \lambda_{3}(t-\tau)+h_{3}\left(e^{i \lambda_{2} t}, e^{i \epsilon=3 / 2 \lambda_{3} t}, e^{i \epsilon \lambda_{3} t}\right) \text {, } \\
& p_{i}=A_{i}+h_{i}\left(e^{i \lambda_{1} t}, e^{i \epsilon / 2 \lambda_{2} t}, e^{i \epsilon \lambda_{3} t}\right)
\end{aligned}
$$

for almost all frequencies near the critical angle, and for sufficiently small $\epsilon$.

\section{REFERENCES}

1. R. B. Barrar, A Proof of the convergence of the Poincare-von Zeipel procedure in celestial mechanics, To appear in The American Journal of Mathematics

2. D. Brouwer, Solution of the problem of artificial satellite theory without drag, Astronomical Journal 64 (1959), pp. 378-397

3. D. Brouwer and G. M. Clemence, Methods of celestial mechanics, Academic Press, 1961

4. C. C. Conley, $A$ disk mapping associated with the satellite problem, Communications on Pure and Applied Mathematics, 17 (1964), pp. 237-243

5. B. Garfinkel, On the motion of a satellite in the vicinity of the critical inclination, The Astronomical Journal 67 (1960), pp. 624-627

6. Y. Hagihara, Libration of an earth satellite with critical inclination, Smithsonian Contributions to Astrophysics 5 (1961), pp. 39-51

7. G. Hori, The motion of an artificial satellite in the vicinity of the critical inclination, The Astronomical Journal 65 (1960), pp. 291-300

8. I. G. Izsak, On the critical inclination in satellite theory, Smithsonian Institution Astrophysical Observatory Special Report No. 90, (1962)

9. Y. Kozai, Motion of a particle with critical inclination in the gravitational field of a spheroid, Smithsonian Contributions to Astrophysics 5 (1961), pp. 53-58

10. W. T. Kyner, Qualitative properties of orbits about an oblate planet, Communications on Pure and Applied Mathematics 17 (1964), pp. 227-236

11. J. Moser, On invariant curves of area-preserving mappings of an annulus, Nachr. Göttingen Math.Phys. Kl., No. 1, 1962

12. H. Poincaré, Les méthodes nouvelles de la mécanique céleste, Vol. I (1892) and Vol. II (1893), Dover Reprint, 1957

13. W. M. Smart, Celestial mechanics, Longmans, Green and Company, Inc., 1953

14. C. L. Siegel, Vorlesungen über Himmelsmechanik, Section 23, Springer-Verlag, Berlin, 1956

15. G. Springer, Introduction to Riemann surfaces, Addison-Wesley Publishing Company, Inc., Reading, Mass., 1957

16. J. P. Vinti, New method of solution for unretarded satellite orbits, Journal of Research of the National Bureau of Standards 63B (1959), pp. 105-116 